\title{
GMR
}

\section{Genotypic correlation and path analysis in early and super-early maize genotypes}

\author{
B.M. Alves and A. Cargnelutti Filho \\ Departamento de Fitotecnia, Universidade Federal de Santa Maria, \\ Santa Maria, RS, Brasil \\ Corresponding author: A. Cargnelutti Filho \\ E-mail: alberto.cargnelutti.filho@gmail.com \\ Genet. Mol. Res. 16 (2): gmr16029671 \\ Received March 16, 2017 \\ Accepted April 26, 2017 \\ Published May 25, 2017 \\ DOI http://dx.doi.org/10.4238/gmr16029671 \\ Copyright (C) 2017 The Authors. This is an open-access article distributed under the terms of \\ the Creative Commons Attribution ShareAlike (CC BY-SA) 4.0 License.
}

\begin{abstract}
The aims of this study were to assess the linear relationships between agronomic and nutritional traits and to identify promising traits for indirect selection in early and super-early maturing maize genotypes. Two trials were run in the 2009/2010 agricultural year, each consisting of a randomized block design with three replications. One trial was run on 36 early maturing maize genotypes and the other on 22 super-early maturing genotypes. Six agronomic traits, 11 protein-nutritional traits, and four energeticnutritional traits were measured. Error normality assumptions were verified using the Kolmogorov-Smirnov test and residual variance homogeneity assumptions using the Levene test. Analysis of variance and the F-test were run for each of the 21 traits. Next, the genotype correlation coefficient matrix was estimated for the 21 traits and each trial. Ridge path analysis was based on values of $\mathrm{k}=0.00$ and $\mathrm{k}=0.10$ on the diagonal of $X^{\prime} X$ correlation matrix, taking the nutritional traits as principal variables and agronomic traits as explanatory variables. The number of days from sowing to male flowering, the number of days from sowing to female flowering, plant height, ear insertion
\end{abstract}

Genetics and Molecular Research 16 (2): gmr16029671 
height, ear weight and grain yield can be used in indirect selection as indicators of grain nutritional quality.

Key words: Zea mays L.; Ridge path analysis; Multicollinearity; Agronomic traits; Nutritional traits; Indirect selection

\section{INTRODUCTION}

Maize (Zea mays L.) is an important crop throughout the world and has both social and economic repercussions. It is widely used for various applications, but mainly as animal feed. Demand for maize, for both human and animal consumption, is expected to increase. Maize breeding researchers seek to combine increased grain yield with improved nutritional quality, especially regarding protein and energetic content. It is, therefore, of fundamental importance to identify the agronomic and nutritional traits of maize genotypes. Maize grains consist of $7.5 \%$ crude protein, $0.24 \%$ lysine, $0.18 \%$ methionine, $0.18 \%$ cysteine, $0.29 \%$ threonine, $0.07 \%$ tryptophan, $0.42 \%$ valine, $0.29 \%$ isoleucine, $1.00 \%$ leucine, $0.42 \%$ phenylalanine, $0.40 \%$ arginine, $3.5 \%$ ether extract, $1.9 \%$ crude fiber (Batal and Dale, 2010), $3438.51 \mathrm{kcal} /$ $\mathrm{kg}$ apparent metabolizable energy corrected for nitrogen ( $\mathrm{Li}$ et al., 2014), and 30\% amylose (Hasjim et al., 2009).

Knowledge of the linear association between agronomic and nutritional maize traits could lead to significant advances in genetic breeding programs, especially when defining crossings, with the aim of targeting animal feed to increase efficiency and cut production costs. Associations between traits can be studied by analyzing a linear correlation coefficient ranging from -1 to 1 . However, this coefficient measures the degree of relatedness between two traits and does not allow direct and indirect influences to be quantified (Cruz et al., 2012).

Path analysis, proposed by Wright (1921), has been used to understand the causes involved in associations between traits. Using this method, it is possible to obtain information on the direct and indirect effects of traits on a principal variable. Path analysis can also be used to identify traits that can be used in indirectly selecting plants, helping researchers to select superior genotypes in agricultural crops such as maize (Bello et al., 2010; Srećkov et al., 2010; Amini et al., 2013; Mustafa et al., 2014; Filipović et al., 2014; Baretta et al., 2016) and soybean (Silva et al., 2015). In addition to conventional path analysis, ridge path analysis allows all traits to be used, provided that a constant $\mathrm{k}$ be added on the diagonal of the matrix correlating the explanatory variables. In ridge path analysis, constant $\mathrm{k}$ ranges from 0 to 1 , and it is recommended to use the lowest k-value capable of stabilizing path coefficients, i.e., working on the degree of multicollinearity between explanatory variables (Cruz et al., 2012).

Ridge path analysis has been used in studies on canola (Coimbra et al., 2005), maize (Carvalho et al., 2001; El-Taweel et al., 2012; Toebe and Cargnelutti Filho, 2013a,b), peppers (Moreira et al., 2013; Silva et al., 2013), bell peppers (Carvalho et al., 1999), and soybean (Bizeti et al., 2004). Studies conducted by Carvalho et al. (1999, 2001), Bizeti et al. (2004), El-Taweel et al. (2012), and Moreira et al. (2013) have confirmed the effectiveness of ridge path analysis in reducing the adverse effects of multicollinearity.

Studies on the linear association between agronomic and nutritional traits using path analysis have been conducted for crops of caster oil (Torres et al., 2015), maize (Zdunić et al., 2012), and soybean (Haghi et al., 2012). These studies show that there is a linear relationship between traits and that it is possible to indirectly select for promising traits in genetic plant

Genetics and Molecular Research 16 (2): gmr16029671 
breeding. However, there are few studies in the literature using ridge path analysis on agronomic and nutritional traits. It is assumed that indirect selection could be used to identify promising agronomic traits for the nutritional quality of maize grains. Since there are no studies that show the real indirect and direct effects of agronomic traits on nutritional traits in the maize crop, the aims of this study were to assess the linear relationships between agronomic and nutritional traits and to identify promising traits for indirect selection in early and super-early maturing maize genotypes.

\section{MATERIAL AND METHODS}

Two trials were run during the 2009/2010 agricultural year on maize (Zea mays L.) crops grown in the experimental area of the Department of Plant Sciences $\left(29^{\circ} 42^{\prime} \mathrm{S}, 53^{\circ} 49^{\prime} \mathrm{W}\right.$ and elevation $95 \mathrm{~m}$ ) of the Federal University of Santa Maria in the Brazilian State of Rio Grande do Sul. Thirty-six early maturing genotypes (trial 1) and 22 super-early maturing genotypes (trial 2) were assessed. The genotypes belonged to the Network of Evaluation of Maize Cultivars of Rio Grande do Sul, coordinated by the State Agricultural Research Foundation (Fundação Estadual de Pesquisa Agropecuária - FEPAGRO).

The trials were designed as randomized block experiments, with three replications. The experimental units consisted of two rows $5 \mathrm{~m}$ long, spaced at $0.80 \mathrm{~m}$ and with plants spaced at $0.20 \mathrm{~m}$ in the row. Seeds were sown manually on October 26, 2009. The plants emerged during the period from November 1st to 3rd, 2009. The plant population was thinned to $62,500 \mathrm{plants} / \mathrm{ha}$. On the day the seeds were sown, basic fertilizer was applied at a rate of $37.5 \mathrm{~kg} /$ ha nitrogen $(\mathrm{N}), 150 \mathrm{~kg} / \mathrm{ha}$ phosphorus $\left(\mathrm{P}_{2} \mathrm{O}_{5}\right)$ and $150 \mathrm{~kg} /$ ha potassium $\left(\mathrm{K}_{2} \mathrm{O}\right)$. Three applications of $200 \mathrm{~kg} / \mathrm{ha} \mathrm{N}$ were applied as topdressing when the plants had developed four, six, and eight expanded leaves, consecutively. The maize ears were harvested on March 15, 2010. The harvests were treated following the technical recommendations for maize.

The following agronomic traits were measured for each field trial, each experimental unit, and each genotype: number of days from sowing to male flowering (MF), number of days from sowing to female flowering (FF; male and female flowering based on $50 \%$ of plants in the plot in flower), plant height on harvesting $(\mathrm{PH}$, in $\mathrm{cm})$, ear insertion height on harvesting $(\mathrm{EH}$, in cm; all plants in the plot were measured for plant height and ear insertion height), ear weight (EW, in $\mathrm{t} / \mathrm{ha}$ ), and grain yield at $13 \%$ moisture content (GY, in t/ha). Then, a $500-\mathrm{g}$ sample of maize grains from each plot was separated. Each sample was placed in a paper bag and heated in a fan oven until the average moisture content was 10\%. After drying, the grains were ground in a micro-mill (MA-630, Marconi) to obtain a particle size between 0.30 and $0.50 \mathrm{~mm}$. After grinding, each sample was placed in a hermetically sealed container pending nutritional analysis. The following protein-nutritional traits were determined for each sample: crude protein (CP), lysine (Lys), methionine (Met), cysteine (Cys), threonine (Thr), tryptophan (Trp), valine (Val), isoleucine (Ile), leucine (Leu), phenylalanine (Phe), and arginine (Arg). The energetic-nutritional traits determined were: apparent metabolizable energy corrected for nitrogen (AMEn, in $\mathrm{kcal} / \mathrm{kg}$ ), ether extract (EE), crude fiber (CF), and amylose (AML), all measured as a percentage of raw material (\%RM). The measurements were taken by nearinfrared reflectance spectroscopy (NIRS), calibrating the spectrometer using the CEAN 010 analytical procedure (Adisseo Brasil SA). Since the NIRS equipment was not calibrated for determining the maize grain amylose content, this trait was determined using the iodometric method described by Martinez and Cuevas-Perez (1989).

Genetics and Molecular Research 16 (2): gmr16029671 
For the agronomic and nutritional traits in each trial, the assumptions of the mathematical model (error normality and homogeneity of residual variances) were tested. Error normality was checked using the Kolmogorov-Smirnov test (Campos, 1983) and homogeneity of residual variances by the Levene test (Steel et al., 1997). Analysis of variance was run, noting the F-test estimate for each genotype $\left(\mathrm{F}_{\mathrm{G}}\right)$, and selective accuracy (SA) determined by the equation: $\mathrm{SA}=\left(1-1 / \mathrm{F}_{\mathrm{G}}\right)^{0.5}$ (Resende and Duarte, 2007). The genotype correlation coefficients $\left(\mathrm{r}_{\mathrm{g}}\right)$ for the 21 traits were determined.

Next, based on the genotype correlation matrix, ridge path analysis under multicollinearity was performed. This analysis involved adding a constant, $\mathrm{k}(\mathrm{k}=0.00$ and $\mathrm{k}=$ $0.10)$, on the diagonal of the $X^{\prime} X$ correlation matrix $(6 \times 6)$ between the explanatory variables (agronomic traits). Ridge path analysis under multicollinearity was carried out based on the genotype correlation coefficients matrix. For each trial, ridge path analysis was performed using the protein-nutritional (CP, Lys, Met, Cys, Thr, Trp, Val, Ile, Leu, Phe, and Arg) and energetic-nutritional (AMEn, EE, CF, and $\mathrm{AML}$ ) traits as the principal variables and the agronomic traits (MF, FF, $\mathrm{PH}, \mathrm{EH}, \mathrm{EW}$, and $\mathrm{GY})$ as explanatory variables, totaling 60 ridge path analysis operations.

The magnitude of multicollinearity was verified using the variance inflation factor(VIF). VIF values below 10 are considered adequate and indicate the absence of multicollinearity, whereas VIF values of 10 or higher indicate a high degree of multicollinearity (Hair et al., 2009). The statistics were analyzed using the GENES program (Cruz, 2013) and Microsoft Office Excel ${ }^{\circledR}$.

\section{RESULTS AND DISCUSSION}

It was verified that $80.95 \%$ (early maturing genotypes) and $95.24 \%$ (super-early maturing genotypes) of the traits measured showed normal distribution, and for $100 \%$ of the traits measured, residual variances were homogeneous for both early maturing and super-early maturing genotypes. Analysis of variance indicated the existence of variability between the early maturing and super-early maturing genotypes for all traits measured. SA was very high $(\mathrm{SA} \geq 0.90)$ for 18 traits, high $(0.70 \leq \mathrm{SA}<0.90)$ for two traits, and low $(0.50 \leq \mathrm{SA}<0.70)$ for one trait in early maturing genotypes. For super-early maturing genotypes, SA was very high $(\mathrm{SA} \geq 0.90)$ for 18 traits and high $(0.70 \leq \mathrm{SA}<0.90)$ for three characters (Table 1$)$.

In overall terms, the results showed that the mathematical assumptions for performing analysis of variance and conducting future studies met the requirements, lending credibility to the trials run.

Estimates of genotype correlation among the 21 traits in early maturing genotypes ranged from $r_{g}=|0.002|$ to $r_{g}=|0.993|$ and in super-early maturing genotypes from $r_{g}=|0.003|$ to $r_{g}=|0.997|$ (Table 2$)$. Generally, in both early maturing and super-early maturing genotypes, the agronomic traits $\mathrm{MF}, \mathrm{FF}, \mathrm{PH}$, and $\mathrm{EH}$ were positively correlated with all protein-nutritional traits. However, EW and GY agronomic traits were negatively correlated with proteinnutritional traits (Table 2).

$\mathrm{MF}, \mathrm{FF}, \mathrm{PH}$, and $\mathrm{EH}$ were positively correlated with AMEn and EE energeticnutritional traits in both early maturing and super-early maturing genotypes and EW and GY were negatively correlated (Table 2). Between MF and CF, and between FF and CF, the correlation was negative in early maturing genotypes and non-existent in super-early maturing genotypes. MF, FF, PH, and EH were negatively correlated with the AML trait in early

Genetics and Molecular Research 16 (2): gmr16029671 
Table 1. Mean, minimum, and maximum values for selective accuracy (SA), error normality, and homogeneity of residual variances between the 21 traits of 36 early maturing maize genotypes and 22 super-early maturing maize genotypes.

\begin{tabular}{|c|c|c|c|c|c|c|c|c|c|c|c|c|}
\hline & \multicolumn{6}{|c|}{ Early } & \multicolumn{6}{|c|}{ Super-early } \\
\hline & Mean & Minimum & Maximum & SA & Z-KS ${ }^{(2)}$ & F-test ${ }^{3}$ & Mean & Minimum & Maximum & SA & Z-KS ${ }^{2}$ & F-test ${ }^{3}$ \\
\hline & \multicolumn{6}{|c|}{ Agronomic } & \multicolumn{6}{|c|}{ Agronomic } \\
\hline $\mathrm{MF}^{\mathrm{I}}$ & 74.28 & 65.00 & 88.00 & 0.94 & $0.615^{\text {ns }}$ & $0.500^{\mathrm{ns}}$ & 72.12 & 66.00 & 82.00 & 0.96 & $0.998^{\mathrm{ns}}$ & $0.220^{\mathrm{ns}}$ \\
\hline$\overline{\mathrm{FF}}$ & 80.19 & 69.00 & 94.00 & 0.97 & $0.655^{\mathrm{ns}}$ & $0.340^{\mathrm{ns}}$ & 77.18 & 69.00 & 87.00 & 0.98 & $0.730^{\mathrm{ns}}$ & $0.260^{\mathrm{ns}}$ \\
\hline $\mathrm{PH}$ & 187.04 & 155.32 & 230.07 & 0.81 & $0.623^{\text {ns }}$ & $0.540^{\mathrm{ns}}$ & 202.27 & 178.15 & 223.51 & 0.91 & $0.491^{\mathrm{ns}}$ & $0.580^{\mathrm{ns}}$ \\
\hline$\overline{\mathrm{EH}}$ & 96.33 & 66.68 & 132.61 & 0.90 & $0.572^{\mathrm{ns}}$ & $0.610^{\mathrm{ns}}$ & 104.97 & 80.08 & 127.20 & 0.96 & $0.750^{\mathrm{ns}}$ & $0.390^{\mathrm{ns}}$ \\
\hline$\overline{\mathrm{EW}}$ & 6.13 & 2.73 & 9.69 & 0.93 & $0.899^{\mathrm{ns}}$ & $0.690^{\mathrm{ns}}$ & 7.27 & 3.97 & 9.89 & 0.92 & $0.559^{\text {ns }}$ & $0.340^{\mathrm{ns}}$ \\
\hline \multirow[t]{2}{*}{$\mathrm{GY}$} & 5.00 & 2.15 & 8.34 & 0.94 & $0.741^{\mathrm{ns}}$ & $0.740^{\mathrm{ns}}$ & 5.92 & 3.09 & 8.23 & 0.94 & $0.669^{\mathrm{ns}}$ & $0.350^{\mathrm{ns}}$ \\
\hline & \multicolumn{6}{|c|}{ Protein-nutritional } & \multicolumn{6}{|c|}{ Protein-nutritional } \\
\hline$\overline{\mathrm{CP}}$ & 7.54 & 6.68 & 8.74 & 0.78 & $0.533^{\mathrm{ns}}$ & $0.380^{\text {ns }}$ & 7.31 & 6.52 & 8.07 & 0.87 & $0.566^{\mathrm{ns}}$ & $0.320^{\text {ns }}$ \\
\hline Lys & 0.21 & 0.20 & 0.23 & 0.90 & $1.679 *$ & $0.720^{\mathrm{ns}}$ & 0.22 & 0.19 & 0.24 & 0.90 & $0.912^{\mathrm{ns}}$ & $0.380^{\mathrm{ns}}$ \\
\hline Met & 0.14 & 0.13 & 0.16 & 0.91 & $2.153^{*}$ & $0.630^{\mathrm{ns}}$ & 0.15 & 0.13 & 0.17 & 0.86 & $1.078^{\mathrm{ns}}$ & $0.410^{\mathrm{ns}}$ \\
\hline Cys & 0.16 & 0.15 & 0.18 & 0.91 & $2.151^{*}$ & $0.580^{\mathrm{ns}}$ & 0.16 & 0.14 & 0.18 & 0.93 & $1.341^{\mathrm{ns}}$ & $0.380^{\mathrm{ns}}$ \\
\hline Thr & 0.21 & 0.17 & 0.25 & 0.90 & $0.871^{\mathrm{ns}}$ & $0.280^{\mathrm{ns}}$ & 0.22 & 0.17 & 0.25 & 0.90 & $0.808^{\mathrm{ns}}$ & $0.320^{\mathrm{ns}}$ \\
\hline Trp & 0.05 & 0.04 & 0.06 & 0.93 & $3.358^{*}$ & $0.710^{\mathrm{ns}}$ & 0.05 & 0.04 & 0.06 & 0.90 & $1.635^{*}$ & $0.480^{\mathrm{ns}}$ \\
\hline Val & 0.30 & 0.26 & 0.35 & 0.95 & $1.225^{\mathrm{ns}}$ & $0.580^{\mathrm{ns}}$ & 0.31 & 0.27 & 0.35 & 0.93 & $0.715^{\mathrm{ns}}$ & $0.560^{\mathrm{ns}}$ \\
\hline Ile & 0.18 & 0.15 & 0.22 & 0.94 & $1.008^{\mathrm{ns}}$ & $0.660^{\mathrm{ns}}$ & 0.19 & 0.15 & 0.22 & 0.95 & $0.971^{\mathrm{ns}}$ & $0.340^{\mathrm{ns}}$ \\
\hline Leu & 0.79 & 0.68 & 0.95 & 0.94 & $0.593^{\mathrm{ns}}$ & $0.420^{\mathrm{ns}}$ & 0.84 & 0.71 & 0.95 & 0.93 & $1.017^{\mathrm{ns}}$ & $0.720^{\text {ns }}$ \\
\hline Phe & 0.27 & 0.23 & 0.33 & 0.96 & $0.954^{\mathrm{ns}}$ & $0.560^{\mathrm{ns}}$ & 0.29 & 0.23 & 0.33 & 0.94 & $0.677^{\mathrm{ns}}$ & $0.710^{\mathrm{ns}}$ \\
\hline \multirow[t]{2}{*}{ Arg } & 0.33 & 0.30 & 0.37 & 0.95 & $0.915^{\mathrm{ns}}$ & $0.490^{\mathrm{ns}}$ & 0.33 & 0.29 & 0.37 & 0.96 & $0.654^{\mathrm{ns}}$ & $0.380^{\mathrm{ns}}$ \\
\hline & \multicolumn{6}{|c|}{ Energetic-nutritional } & \multicolumn{6}{|c|}{ Energetic-nutritional } \\
\hline AMEn & 3434.87 & 3382.00 & 3479.00 & 0.93 & $0.624^{\mathrm{ns}}$ & $0.540^{\mathrm{ns}}$ & 3416.05 & 3332.00 & 3473.00 & 0.98 & $0.396^{\mathrm{ns}}$ & $0.560^{\mathrm{ns}}$ \\
\hline EE & 3.76 & 3.37 & 4.18 & 0.93 & $0.674^{\mathrm{ns}}$ & $0.660^{\mathrm{ns}}$ & 3.72 & 3.21 & 4.09 & 0.96 & $0.875^{\text {ns }}$ & $0.870^{\text {ns }}$ \\
\hline $\mathrm{CF}$ & 1.97 & 1.58 & 2.45 & 0.33 & $0.521^{\mathrm{ns}}$ & $0.540^{\mathrm{ns}}$ & 1.92 & 1.57 & 2.18 & 0.75 & $0.519^{\mathrm{ns}}$ & $0.400^{\text {ns }}$ \\
\hline AML & 28.96 & 26.83 & 31.47 & 0.90 & $0.887^{\mathrm{ns}}$ & $0.450^{\mathrm{ns}}$ & 28.61 & 26.04 & 30.68 & 0.92 & $0.776^{\mathrm{ns}}$ & $0.440^{\mathrm{ns}}$ \\
\hline
\end{tabular}

${ }^{1}$ Agronomic traits: $\mathrm{MF}=$ number of days from sowing until male flowering; $\mathrm{FF}=$ number of days from sowing until female flowering; $\mathrm{PH}=$ plant height, in cm; $\mathrm{EH}=$ ear insertion height, in $\mathrm{cm} ; \mathrm{EW}=$ ear weight, in $\mathrm{t} / \mathrm{ha}$; $\mathrm{GY}=$ grain yield, in $\mathrm{t} / \mathrm{ha}$; protein-nutritional traits: $\mathrm{CP}=$ crude protein, in $\% \mathrm{RM}$; Lys $=$ lysine, in $\% \mathrm{RM}$; Met $=$ methionine, in \%RM; Cys = cysteine, in \%RM; Thr = threonine, in \%RM; Trp = tryptophan, in \%RM; Val = valine, in \%RM; Ile = isoleucine, in \%RM; Leu = leucine, in \%RM; Phe = phenylalanine, in \%RM; Arg = arginine, in \%RM; and energetic-nutritional traits: AMEn = apparent metabolizable energy corrected for nitrogen, in $\mathrm{kcal} /$ $\mathrm{kg} ; \mathrm{EE}=$ ether extract, in \% $\mathrm{RM} ; \mathrm{CF}=$ crude fiber, in \% $\mathrm{RM}$; $\mathrm{AML}=$ amylose, in \% $\mathrm{RM} .{ }^{2}$ Kolmogorov Smirnov test values for the normality of the error distribution. ${ }^{n}$ Nonsignificant (normal distribution). ${ }^{3}$ Levene test values for the

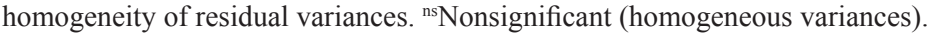

maturing genotypes and positively correlated in super-early maturing genotypes. Generally, in both early maturing and super-early maturing genotypes, the correlation between the agronomic traits EW and GY and energetic-nutritional traits was negative. However, CF was positively correlated with EW $\left(r_{g}=0.594\right)$ and $\mathrm{GY}\left(r_{\mathrm{g}}=0.594\right)$ in early maturing genotypes. In super-early maturing genotypes, there was no correlation between $\mathrm{EW}$ and $\mathrm{CF}\left(\mathrm{r}_{\mathrm{g}}=0.008\right)$, and between GY and CF ( $\left.\mathrm{r}_{\mathrm{g}}=0.053\right)$ (Table 2). These genotype correlation estimates can be used to verify relationships between agronomic and nutritional traits (protein and energetic) and facilitate improvements in given traits using indirect selection.

The results indicate that GY was negatively correlated with protein-nutritional traits in both early maturing and super-early maturing genotypes. Other studies on maize crops have also reported a negative correlation between GY and CP (Idikut et al., 2009; Aliu et al., 2012; Mahesh et al., 2013; Bekele and Rao, 2014), and between GY and grain amino acid composition (Lovatto et al., 2006); this shows that it is not possible to select plants with high grain yield and concomitant high protein quality.

In early maturing genotypes, the correlations between $\mathrm{MF}$ and $\mathrm{EE}$, and between $\mathrm{FF}$ and $\mathrm{EE}$ were positive, with respective values of $\mathrm{r}_{\mathrm{g}}=0.241$ and $\mathrm{r}_{\mathrm{g}}=0.342$. For super-early 
Table 2. Estimates of genotype correlation coefficients between the 21 traits of 36 early maturing maize genotypes (above the diagonal) and 22 super-early maturing maize genotype (below the diagonal).

\begin{tabular}{|c|c|c|c|c|c|c|c|c|c|c|c|}
\hline Trait $^{1}$ & MF & FF & $\mathrm{PH}$ & EH & EW & GY & $\mathrm{CP}$ & Lys & Met & Cys & Thr \\
\hline MF & 1 & 0.875 & 0.263 & 0.446 & -0.579 & -0.554 & 0.035 & 0.377 & 0.342 & 0.384 & 0.435 \\
\hline FF & 0.900 & 1 & 0.279 & 0.256 & -0.733 & -0.741 & 0.020 & 0.537 & 0.412 & 0.473 & 0.577 \\
\hline PH & 0.523 & 0.501 & 1 & 0.464 & 0.020 & 0.029 & 0.280 & 0.289 & 0.101 & 0.457 & 0.020 \\
\hline EH & 0.853 & 0.805 & 0.655 & 1 & -0.030 & -0.002 & 0.562 & 0.367 & 0.191 & 0.452 & 0.159 \\
\hline EW & -0.530 & -0.733 & -0.181 & -0.530 & 1 & 0.993 & -0.169 & -0.562 & -0.321 & -0.524 & -0.741 \\
\hline$\overline{\mathrm{GY}}$ & -0.545 & -0.765 & -0.186 & -0.533 & 0.992 & 1 & -0.185 & -0.619 & -0.354 & -0.575 & -0.783 \\
\hline CP & 0.334 & 0.377 & -0.134 & 0.073 & -0.359 & -0.361 & 1 & 0.383 & -0.023 & 0.486 & 0.273 \\
\hline Lys & 0.368 & 0.507 & -0.003 & 0.266 & -0.706 & -0.722 & 0.640 & 1 & 0.760 & 0.902 & 0.948 \\
\hline Met & 0.571 & 0.735 & 0.283 & 0.552 & -0.684 & -0.709 & 0.388 & 0.832 & 1 & 0.643 & 0.769 \\
\hline Cys & 0.620 & 0.760 & 0.106 & 0.427 & -0.785 & -0.793 & 0.781 & 0.856 & 0.751 & 1 & 0.801 \\
\hline Thr & 0.606 & 0.740 & 0.101 & 0.377 & -0.849 & -0.857 & 0.686 & 0.950 & 0.911 & 0.966 & 1 \\
\hline Trp & 0.581 & 0.639 & -0.056 & 0.384 & -0.852 & -0.825 & 0.654 & 0.962 & 0.829 & 0.962 & 0.961 \\
\hline Val & 0.542 & 0.723 & 0.177 & 0.430 & -0.870 & -0.870 & 0.575 & 0.956 & 0.956 & 0.947 & 0.965 \\
\hline Ile & 0.519 & 0.712 & 0.156 & 0.355 & -0.861 & -0.852 & 0.612 & 0.898 & 0.932 & 0.937 & 0.954 \\
\hline Leu & 0.478 & 0.640 & 0.048 & 0.291 & -0.878 & -0.869 & 0.570 & 0.846 & 0.799 & 0.919 & 0.867 \\
\hline Phe & 0.545 & 0.684 & 0.073 & 0.363 & -0.841 & -0.835 & 0.653 & 0.896 & 0.876 & 0.973 & 0.954 \\
\hline Arg & 0.559 & 0.674 & 0.137 & 0.455 & -0.682 & -0.708 & 0.664 & 0.935 & 0.930 & 0.971 & 0.991 \\
\hline AMEn & 0.444 & 0.523 & 0.429 & 0.645 & -0.152 & -0.238 & 0.178 & 0.522 & 0.833 & 0.469 & 0.559 \\
\hline EE & 0.683 & 0.725 & 0.355 & 0.721 & -0.377 & -0.442 & 0.353 & 0.668 & 0.846 & 0.687 & 0.754 \\
\hline$\overline{\mathrm{CF}}$ & 0.047 & 0.005 & 0.335 & 0.048 & 0.008 & 0.053 & -0.564 & -0.349 & -0.315 & -0.270 & -0.403 \\
\hline \multirow[t]{2}{*}{ AML } & 0.239 & 0.319 & 0.206 & 0.427 & -0.141 & -0.196 & -0.111 & -0.105 & 0.249 & -0.055 & 0.054 \\
\hline & Trp & Val & Ile & Leu & Phe & Arg & AMEn & EE & CF & AML & \\
\hline MF & 0.434 & 0.340 & 0.355 & 0.220 & 0.324 & 0.351 & 0.228 & 0.241 & -0.679 & -0.332 & \\
\hline FF & 0.558 & 0.513 & 0.543 & 0.408 & 0.498 & 0.450 & 0.207 & 0.342 & -0.834 & -0.402 & \\
\hline PH & 0.058 & 0.284 & 0.110 & 0.265 & 0.249 & 0.290 & 0.139 & 0.450 & 0.140 & -0.527 & \\
\hline EH & 0.143 & 0.257 & 0.106 & 0.188 & 0.200 & 0.452 & 0.511 & 0.398 & 0.008 & -0.049 & \\
\hline EW & -0.648 & -0.547 & -0.626 & -0.551 & -0.581 & -0.614 & -0.339 & -0.153 & 0.594 & 0.262 & \\
\hline GY & -0.710 & -0.607 & -0.677 & -0.612 & -0.635 & -0.648 & -0.324 & -0.207 & 0.594 & 0.273 & \\
\hline$\overline{\mathrm{CP}}$ & 0.348 & 0.402 & 0.220 & 0.464 & 0.325 & 0.618 & 0.155 & 0.408 & -0.045 & -0.333 & \\
\hline Lys & 0.943 & 0.844 & 0.838 & 0.728 & 0.869 & 0.916 & 0.487 & 0.607 & -0.200 & -0.547 & \\
\hline Met & 0.741 & 0.758 & 0.810 & 0.577 & 0.802 & 0.730 & 0.533 & 0.468 & -0.318 & -0.237 & \\
\hline Cys & 0.970 & 0.946 & 0.875 & 0.841 & 0.919 & 0.944 & 0.314 & 0.743 & 0.259 & -0.530 & \\
\hline Thr & 0.979 & 0.908 & 0.944 & 0.806 & 0.941 & 0.928 & 0.347 & 0.435 & 0.218 & -0.333 & \\
\hline Trp & 1 & 0.964 & 0.931 & 0.877 & 0.961 & 0.916 & 0.290 & 0.654 & 0.463 & -0.368 & \\
\hline Val & 0.987 & 1 & 0.968 & 0.915 & 0.987 & 0.912 & 0.226 & 0.561 & 0.166 & -0.439 & \\
\hline Ile & 0.950 & 0.994 & 1 & 0.862 & 0.970 & 0.867 & 0.207 & 0.486 & 0.257 & -0.350 & \\
\hline Leu & 0.908 & 0.970 & 0.953 & 1 & 0.939 & 0.805 & 0.090 & 0.333 & 0.349 & -0.375 & \\
\hline Phe & 0.978 & 0.997 & 0.987 & 0.975 & 1 & 0.898 & 0.242 & 0.445 & 0.373 & -0.407 & \\
\hline Arg & 0.965 & 0.964 & 0.928 & 0.867 & 0.952 & 1 & 0.525 & 0.628 & 0.043 & -0.392 & \\
\hline AMEn & 0.441 & 0.534 & 0.449 & 0.328 & 0.448 & 0.664 & 1 & 0.536 & -0.065 & -0.105 & \\
\hline EE & 0.680 & 0.695 & 0.635 & 0.509 & 0.636 & 0.806 & 0.945 & 1 & -0.480 & -0.415 & \\
\hline$\overline{\mathrm{CF}}$ & -0.304 & -0.326 & -0.294 & -0.310 & -0.402 & -0.480 & -0.413 & -0.405 & 1 & 0.737 & \\
\hline AML & -0.069 & -0.006 & -0.025 & -0.111 & -0.066 & 0.082 & 0.645 & 0.434 & -0.660 & 1 & \\
\hline
\end{tabular}

${ }^{1}$ Agronomic traits: $\mathrm{MF}=$ number of days from sowing until male flowering; $\mathrm{FF}=$ number of days from sowing until female flowering; $\mathrm{PH}=$ plant height, in $\mathrm{cm} ; \mathrm{EH}=$ ear insertion height, in $\mathrm{cm} ; \mathrm{EW}=$ ear weight, in $\mathrm{t} / \mathrm{ha} ; \mathrm{GY}=$ grain yield, in t/ha; protein-nutritional traits: $\mathrm{CP}=$ crude protein, in $\% \mathrm{RM}$; Lys $=$ lysine, in $\% \mathrm{RM}$; Met = methionine, in $\% \mathrm{RM}$; Cys = cysteine, in \%RM; Thr = threonine, in \%RM; Trp = tryptophan, in $\% \mathrm{RM}$; Val = valine, in \%RM; $\mathrm{Ile}=$ isoleucine, in \%RM; Leu = leucine, in \%RM; Phe = phenylalanine, in \%RM; Arg = arginine, in \%RM; and energetic-nutritional traits: $\mathrm{AMEn}=$ apparent metabolizable energy corrected for nitrogen, in $\mathrm{kcal} / \mathrm{kg} ; \mathrm{EE}=$ ether extract, in \% $\mathrm{RM} ; \mathrm{CF}=$ crude fiber, in \% $\mathrm{RM} ; \mathrm{AML}=$ amylose, in \% $\mathrm{RM}$.

maturing genotypes, the correlation of MF and FF with EE was also positive and of higher magnitude compared to the early maturing genotypes, with respective values of $r_{g}=0.683$ and $r_{\mathrm{g}}=0.725$. A low-magnitude positive correlation between these traits was also reported by Wali et al. (2006) and Chukwu et al. (2015). The correlations between GY and EE were $\mathrm{r}_{\mathrm{g}}$ $=-0.207$ and $r_{g}=-0.442$, respectively, for early maturing and super-early maturing genotypes. Studies conducted by Fabijanac et al. (2006) and Saleem et al. (2008) also reported negative correlations between these traits in maize; this leads us to infer that higher values of MF and 
FF result in higher EE, and higher GY results in lower EE in both early maturing and superearly maturing genotypes.

Ridge path analysis with constant $\mathrm{k}=0.00$ showed the presence of multicollinearity. Respective VIF values were $0.000 \leq \mathrm{VIF} \leq 111.483$ and $0.068 \leq \mathrm{VIF} \leq 110.278$ for both early maturing and super-early maturing genotypes (Tables 3 and 4 ).

Table 3. Estimates of genotypic correlation coefficients and respective estimates of the direct and indirect effect of the explanatory variables based on ridge path analysis performed using values of $\mathrm{k}=0.00$ and path analysis, determination coefficient, and effect of the residual variable for the 36 early maturing maize genotypes.

\begin{tabular}{|c|c|c|c|c|c|c|c|c|c|c|c|c|c|c|c|c|}
\hline \multirow[t]{3}{*}{$\overline{\text { Effect }}$} & \multicolumn{16}{|c|}{ Main variable } \\
\hline & & & & & & in-nutrii & & & & & & & Energetic- & nutrition: & & \\
\hline & $\mathrm{CP}$ & Lys & Met & Cys & Thr & $\operatorname{Trp}$ & Val & Ile & Leu & Phe & Arg & AMEn & $\mathrm{EE}$ & $\mathrm{CF}$ & AML & $\mathrm{VIF}^{1}$ \\
\hline Direct of MF on & 0.087 & 0.237 & 0.250 & 0.751 & 0.438 & 0.892 & 0.326 & 0.212 & 0.332 & 0.325 & 0.288 & -0.265 & 0.159 & 0.687 & -0.510 & 8.716 \\
\hline Indirect of MF via FF on & -0.816 & -0.438 & -0.033 & -1.105 & -0.554 & -0.918 & -0.509 & -0.281 & -0.758 & -0.547 & -0.760 & -0.128 & -0.181 & -1.721 & 0.294 & 9.226 \\
\hline Indirect of MF via PH on & $\begin{array}{c}-0.010 \\
0.060\end{array}$ & $\begin{array}{c}-0.450 \\
0.065\end{array}$ & $\begin{array}{l}-0.003 \\
0.001\end{array}$ & $\frac{-1.103}{0.141}$ & 0.014 & $\begin{array}{ll}-0.910 \\
0.040\end{array}$ & 0.083 & $\begin{array}{l}-0.201 \\
0.037\end{array}$ & $\begin{array}{c}-0.150 \\
0.104\end{array}$ & 0.084 & 0.075 & $\begin{array}{ll}-0.120 \\
-0.014\end{array}$ & $\begin{array}{l}-0.101 \\
0.094\end{array}$ & $\frac{-1.121}{0.141}$ & $\begin{array}{c}-0.178 \\
-0.178\end{array}$ & $\frac{9.220}{0.110}$ \\
\hline Indirect of MF via EH on & 0.326 & 0.183 & 0.073 & 0.156 & 0.091 & 0.047 & 0.112 & 0.059 & 0.097 & 0.085 & 0.229 & 0.302 & 0.152 & 0.010 & 0.165 & 0.350 \\
\hline Indirect of MF via EW on & -1.647 & $\frac{0.105}{-2.961}$ & $\frac{0.073}{-1.656}$ & $\frac{.150}{-3.447}$ & $\frac{-2.240}{-2.240}$ & -3.548 & -3.009 & -2.301 & $\frac{.0 .068}{-3.068}$ & -2.720 & -2.200 & 0.268 & $\frac{0.152}{-2.755}$ & -1.411 & 0.833 & 33.723 \\
\hline Indirect of MF via GY on & 2.025 & 3.291 & 1.707 & 3.888 & 2.687 & 3.921 & 3.337 & 2.629 & 3.512 & 3.096 & 2.720 & 0.065 & 2.772 & 1.616 & -0.935 & 34.181 \\
\hline Genotypic correlation $\left(\mathrm{r}_{\mathrm{g}}\right)$ & 2.0235 & 0.377 & 0.342 & 3.0000 & 0.035 & 0.434 & 0.340 & 20.355 & $\frac{0.122}{0.220}$ & 0.324 & $\frac{2.120}{0.351}$ & 0.228 & 0.241 & 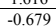 & $\begin{array}{l}-0.933 \\
-0.332\end{array}$ & \\
\hline Direct of FF on & -0.932 & -0.501 & -0.038 & -1.263 & -0.634 & -1.049 & -0.582 & -0.322 & -0.866 & -0.625 & -0.869 & -0.146 & -0.207 & -1.968 & 0.336 & 12.053 \\
\hline Indirect of $\mathrm{FF}$ via $\mathrm{MF}$ on & 0.076 & 0.207 & 0.219 & 0.657 & 0.383 & 0.780 & 0.285 & 0.185 & 0.291 & 0.285 & 0.252 & -0.232 & 0.139 & 0.601 & -0.446 & 6.672 \\
\hline dire & 0.064 & 0.069 & 0.001 & 0.150 & 0.015 & 0.043 & 0.088 & 0.040 & 0.111 & 0.089 & 0.080 & -0.015 & 0.100 & 0.150 & -0.189 & 0.125 \\
\hline Indirect of FF via $\mathrm{EH}$ on & 0.187 & 0.105 & 0.042 & 0.090 & 0.052 & 0.027 & 0.064 & 0.034 & 0.056 & 0.049 & 0.131 & 0.174 & 0.088 & 0.006 & 0.095 & 0.115 \\
\hline Indirect of FF via EW on & -2.086 & -3.749 & -2.097 & -4.365 & -2.836 & -4.492 & -3.809 & -2.913 & -3.885 & -3.444 & -2.785 & 0.339 & -3.488 & -1.787 & 1.054 & 54.057 \\
\hline Indirect of FF via GY on & $\frac{-2.000}{2.711}$ & $\frac{-3.149}{4.405}$ & 2.285 & - 5.0 .003 & $\frac{-2.050}{3.597}$ & $\begin{array}{ll}-\frac{3.424}{5.248} \\
\end{array}$ & 4.466 & 3.519 & 4.000 & $\frac{-3.444}{4.144}$ & $\frac{-2.100}{3.640}$ & 0.087 & 3.710 & $\frac{-1.101}{2.163}$ & $\frac{1.004}{-1.251}$ & $\frac{34.057}{61.226}$ \\
\hline & 0.020 & 0.537 & 0.412 & 0.473 & 0.577 & 0.558 & 0.51 & 0.543 & 0.408 & 0.49 & 0.450 & 0.2 & 0.342 & -0.8 & -0.402 & \\
\hline Direct of $\mathrm{PH}$ on & 0.228 & 0.248 & 0.002 & 0.537 & 0.052 & 0.152 & 0.317 & 0.142 & 0.398 & 0.319 & 0.286 & -0.052 & 0.358 & 0.535 & $\begin{array}{l}-0.4678 \\
-0.678\end{array}$ & 1.598 \\
\hline Indirect of $\mathrm{PH}$ via MF on & 0.023 & 0.062 & 0.066 & 0.197 & 0.115 & 0.234 & 0.086 & 0.056 & 0.087 & 0.085 & 0.076 & -0.070 & 0.042 & 0.180 & -0.134 & 0.601 \\
\hline Indirect of $\mathrm{PH}$ via FF on & $\frac{0.023}{-0.260}$ & $\frac{-0.02}{-0.140}$ & $\frac{0.000}{-0.010}$ & -0.1353 & $\frac{-11177}{-0.177}$ & -0.293 & -0.0003 & $\frac{0.050}{-0.090}$ & $\frac{-0.017}{-0.242}$ & $\frac{0.035}{-0.175}$ & -0.0243 & $\begin{array}{ll}-0.070 \\
-0.041\end{array}$ & $\frac{0.042}{-0.058}$ & $\frac{-.100}{-0.550}$ & $\begin{array}{l}-0.1 .094 \\
0.094\end{array}$ & 0.091 \\
\hline & 0.339 & 0.190 & 0.076 & 0.162 & 0.094 & 0.049 & 0.117 & 0.062 & 0.101 & 0.088 & 0.238 & 0.3 & 0.158 & 0.0 & & 0.377 \\
\hline Indirect of $\mathrm{PH}$ via $\mathrm{EW}$ on & 0.056 & 0.101 & 0.057 & 0.118 & 0.077 & 0.121 & 0.103 & 0.079 & 0.105 & 0.093 & 0.075 & -0.009 & 0.094 & 0.048 & -0.028 & 0.040 \\
\hline Indirect of PH via GY on & -0.106 & -0.172 & -0.089 & -0.204 & -0.141 & -0.205 & -0.175 & -0.138 & -0.184 & -0.162 & -0.142 & -0.003 & -0.145 & -0.085 & 0.049 & 0.089 \\
\hline Genotypic correlation $\left(\mathrm{r}_{\mathrm{g}}\right)$ & 0.280 & 0.289 & 0.101 & 0.457 & 0.020 & 0.058 & 0.284 & 0.110 & 0.265 & 0.249 & 0.290 & 0.139 & 0.450 & 0.140 & -0.527 & \\
\hline & 0.731 & 0.410 & 0.16 & 0.350 & 0.2 & & 0.251 & 0.133 & 0.217 & 0.191 & 0.513 & 0.6 & 0.342 & 0.0 & 0.369 & 1.756 \\
\hline via MF on & 0.039 & 0.106 & 0.1112 & 0.335 & 0.195 & 0.398 & 0.146 & 0.094 & 0.148 & 0.145 & 0.128 & $\frac{0.010}{-0.118}$ & $\frac{0.342}{0.071}$ & 0.306 & -0.228 & $\begin{array}{l}1.730 \\
1.734\end{array}$ \\
\hline FF on & -0.239 & -0.128 & -0.010 & -0.324 & -0.162 & -0.269 & -0.149 & -0.082 & -0.222 & -0.160 & -0.223 & -0.037 & -0.053 & -0.504 & 0.086 & 0.792 \\
\hline direc & 0.106 & 0.115 & 0.001 & 0.249 & 0.024 & 0.071 & 0.147 & 0.066 & 0.184 & 0.148 & 0.133 & -0.024 & 0.166 & 0.248 & -0.314 & 0.343 \\
\hline dir & -0.0 & -0.151 & -0.0 & -0.176 & -0.1 & & -0.1 & -0.117 & -0.1 & -0.1 & -0 . & 0.0 & -0.140 & -0.0 & & 0.091 \\
\hline Indirect & 0.009 & 0.014 & 0.007 & 0.017 & 0.012 & 0.017 & 0.014 & 0.011 & 0.015 & 0.013 & 0.012 & 0.000 & 0.012 & 0.007 & $\frac{-0.042}{-0.004}$ & 0.000 \\
\hline Genoty & 0.562 & 0.367 & 0.191 & 0.452 & 0.159 & 0.143 & 0.257 & 0.106 & 0.188 & 0.200 & 0.452 & 0.5 & 0.398 & 0.008 & -0.049 & \\
\hline Direct o & 2.8 & & 2.862 & 5.9 & & 6.1 & & & & 4.7 & 3.8 & -0.462 & 4.760 & 2.4 & -1.439 & 100.665 \\
\hline & .050 & -0.137 & -0.145 & -0.434 & -0.2 & & -0.18 & -0.122 & -0.19 & -0.1 & -0.1 & 0.1 & -0.092 & -0.3 & & 2.920 \\
\hline & 0.683 & 0.367 & 0.027 & 0.926 & 0.464 & 0.769 & 0.42 & 0.236 & 0.635 & 0.4 & 0.63 & 0.11 & 0.152 & 1.4 & $\frac{-.246}{-0.246}$ & 6.472 \\
\hline Indire & 0.00 & 0.005 & 0.007 & 0.911 & $\begin{array}{l}0.40 \\
0.00\end{array}$ & 0.06 & 0.006 & 0.003 & 0.008 & 0.0 & 0.006 & -0.001 & 0.007 & 0.0 & $\begin{array}{l}-0.24013 \\
-0.013\end{array}$ & $\begin{array}{l}0.4 / 2 \\
0.001\end{array}$ \\
\hline Indirect & -0.0 & $\begin{array}{c}-0.012 \\
\end{array}$ & & -0.0 & & & & -0.004 & -0.006 & -0.0 & & & -0.010 & -0.0 & -0.011 & 0.002 \\
\hline$\frac{\mathrm{min}}{\mathrm{In}}$ & $\begin{array}{l}-0.022 \\
-3.630\end{array}$ & $\begin{array}{l}-0.012 \\
-5.899\end{array}$ & $\begin{array}{l}-0.003 \\
-3.060\end{array}$ & $\frac{-0.010}{-6.970}$ & $\begin{array}{l}-0.00 \\
-4.81\end{array}$ & $\begin{array}{l}-0.003 \\
-7.028\end{array}$ & $\begin{array}{l}-0.001 \\
-5.981\end{array}$ & $\begin{array}{l}-0.004 \\
-4.712\end{array}$ & $\begin{array}{l}-0.000 \\
-6.295\end{array}$ & $\begin{array}{l}-0.04 \\
-5.5\end{array}$ & & $\frac{-0.6}{-0.1}$ & $\begin{array}{l}-0.0100 \\
-4.968\end{array}$ & $\frac{-0.0}{-2.8}$ & $\frac{-0.011}{1.676}$ & $\begin{array}{l}0.002 \\
109.822\end{array}$ \\
\hline Genotypic correlation $\left(\mathrm{rg}_{\mathrm{g}}\right)$ & -0.169 & -0.562 & -0.321 & -0.524 & -0.741 & -0.648 & -0.54 & -0.626 & -0.551 & -0.5 & -0.614 & -0.3 & -0.153 & 0.5 & 0.262 & \\
\hline Direct & -3.6 & -5.944 & & -7.022 & & -7.0 & & -4.748 & & -5.5 & & -0.1 & -5.006 & & 1.688 & 111.483 \\
\hline Indirs & -0.048 & -0.131 & -0.138 & -0.416 & -0.242 & -0.494 & -0.0281 & -0.117 & -0.184 & -0.180 & -0.159 & 0.147 & -0.088 & -0.380 & 0.282 & 2.672 \\
\hline Indirect of GY via FF on & 0.691 & 0.371 & 0.028 & 0.936 & 0.470 & 0.777 & 0.431 & 0.238 & 0.642 & 0.463 & 0.644 & 0.108 & 0.153 & 1.458 & -0.249 & 6.619 \\
\hline & & 0.007 & 0.000 & 0.016 & 0.002 & & 0.009 & 0.004 & 0.012 & 0.00 & 0.008 & -0.002 & & 0.016 & -0.020 & 0.001 \\
\hline & -0.0 & -0.0 & & -0.0 & 0.0 & & & 0.000 & -0.0 & & -0.0 & & -0.001 & 0.000 & -0.001 & 0.000 \\
\hline Indirect of GY via EW on & 2.025 & 5.077 & 2.840 & 5.912 & 3.842 & 6.084 & 5.159 & 3.946 & 5.261 & 4.665 & 3.772 & -0.459 & 4.724 & 2.420 & -1.428 & 99.165 \\
\hline Genotypic correlation $\left(\mathrm{r}_{\mathrm{g}}\right)$ & $\frac{2.825}{-0.185}$ & $\frac{5.0719}{-0.619}$ & $\frac{2.040}{-0.354}$ & $\begin{array}{c}-5.912 \\
-0.575\end{array}$ & $\frac{-0.042}{-0.783}$ & $\frac{0.084}{-0.710}$ & $\begin{array}{c}-0.1597 \\
-0.607\end{array}$ & $\begin{array}{c}3.940 \\
-0.677\end{array}$ & $\frac{0.201}{-0.612}$ & - -4.0035 & $\frac{3.772}{-0.648}$ & $\begin{array}{l}-0.459 \\
-0.324\end{array}$ & $\frac{4.124}{-0.207}$ & $\frac{2.420}{0.594}$ & $\begin{array}{c}-1.428 \\
0.273\end{array}$ & \\
\hline & & & & & & & & & & & & & & 0.964 & & \\
\hline $\begin{array}{l}\text { Determination coe } \\
\text { Residual variable }\end{array}$ & $\begin{array}{l}0.653 \\
0.588\end{array}$ & 0.838 & 0.252 & $\frac{1.0014}{0.000}$ & 0.457 & 0.822 & $\frac{0.797}{0.470}$ & 0.586 & 0.0 .421 & $\begin{array}{l}0.153 \\
0.517\end{array}$ & 0.358 & 0.043 & 0.655 & 0.184 & 0.737 & \\
\hline
\end{tabular}

${ }^{1} \mathrm{VIF}=$ variance inflation factor. Number of days from sowing until male flowering (MF), number of days from sowing until female flowering (FF), plant height (PH), ear insertion height (EH), ear weight (EW), and grain yield $(\mathrm{GY})$ on the main variables: crude protein (CP), lysine (Lys), methionine (Met), cysteine (Cys), threonine (Thr), tryptophan (Trp), valine (Val), isoleucine (Ile), leucine (Leu), phenylalanine (Phe), and arginine (Arg), apparent metabolizable energy corrected for nitrogen (AMEn), ether extract (EE), crude fiber (CF), and amylose (AML).

VIF $\geq 10$ confirms the presence of multicollinearity in the explanatory variable correlation matrix (Hair et al., 2009). Ridge path analysis $(\mathrm{k}=0.00)$ overestimated the path coefficient values (direct and indirect effects), with wide variability and, therefore, it was not possible to make appropriate inferences in early maturing and super-early maturing genotypes. Studies based on ridge path analysis $(\mathrm{k}=0.00)$ in maize crops by Carvalho et al. $(2001)$ and El-Taweel et al. (2012), and in soybean by Bizeti et al. (2004) also reported that ridge path analysis correlations were violated.

Genetics and Molecular Research 16 (2): gmr16029671 


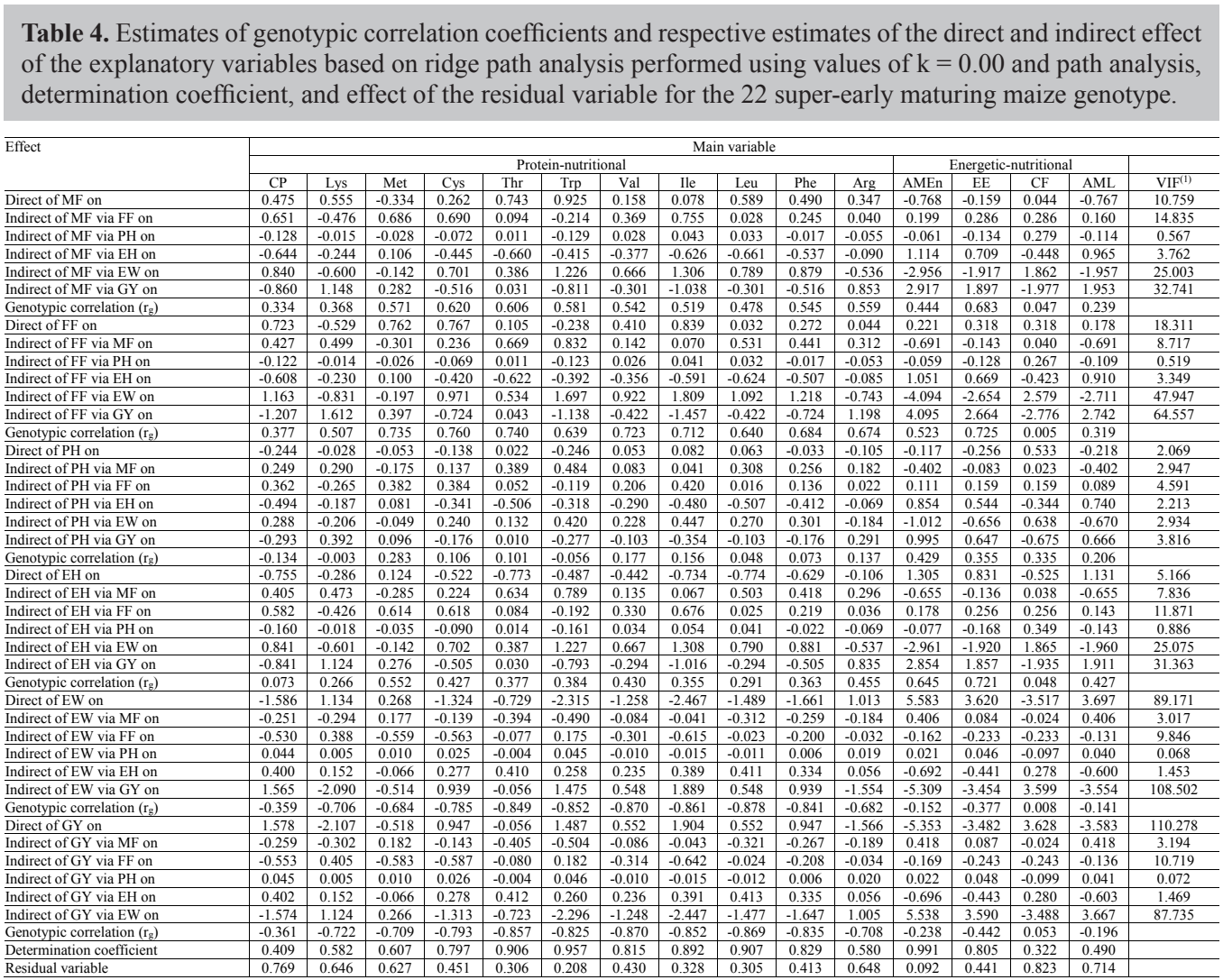

${ }^{1} \mathrm{VIF}=$ variance inflation factor. Number of days from sowing until male flowering (MF), number of days from sowing until female flowering $(\mathrm{FF})$, plant height $(\mathrm{PH})$, ear insertion height $(\mathrm{EH})$, ear weight $(\mathrm{EW})$, and grain yield (GY) on the main variables: crude protein (CP), lysine (Lys), methionine (Met), cysteine (Cys), threonine (Thr), tryptophan (Trp), valine (Val), isoleucine (Ile), leucine (Leu), phenylalanine (Phe), and arginine (Arg), apparent metabolizable energy corrected for nitrogen (AMEn), ether extract (EE), crude fiber (CF), and amylose (AML).

To get around this problem of multicollinearity in the correlation matrix, a constant, $\mathrm{k}$ $=0.10$, was added on the diagonal of the $\mathrm{X}^{\prime} \mathrm{X}$ matrix, resulting in VIF values below ten in the correlation matrices for early maturing genotypes $(\mathrm{VIF} \leq 6.415)$ and for super-early maturing genotypes (VIF $\leq 6.556$ ), indicating the absence of multicollinearity (Tables 5 and 6 ).

Introducing $\mathrm{k}=0.10$ overcomes correlation matrix multicollinearity problems so that biological inferences can be made for both early maturing and super-early maturing genotypes.

Tables 5 and 6 indicate the direct and indirect effects, via agronomic traits, on proteinnutritional and energetic-nutritional traits for a $\mathrm{k}$-value of $\mathrm{k}=0.10$. Based on the genotype correlation coefficient matrix, for early maturing genotypes, the FF trait showed a negative linear correlation $\left(r_{\sigma}=-0.834\right)$ and a direct negative effect (direct effect $\left.=-0.821\right)$ on CF. PH showed a negative linear correlation $\left(\mathrm{r}_{\mathrm{g}}=-0.527\right)$ and a direct negative effect (direct effect $=$ $-0.536)$ on AML. The EH trait showed a positive linear correlation and a positive direct effect on $\mathrm{CP}$ (direct effect $=0.635)$ and AMEn (direct effect $=0.565)$. GY showed a negative linear correlation $\left(-0.783 \leq \mathrm{r}_{\mathrm{g}} \leq-0.575\right)$ with the protein-nutritional traits Lys, Cys, Thr, Trp, Val, Ile, 
Leu, Phe, and Arg, and a direct negative effect $(-0.619 \leq$ direct effect $\leq-0.501)$ with the same sign and similar magnitude, confirming the cause and effect relationship between GY and Lys, Cys, Thr, Trp, Val, Ile, Leu, Phe, and Arg. The direct effects of MF, FF, and EW on Lys, Cys, Thr, Trp, Val, Ile, Leu, Phe, and Arg were negligible and, therefore, the existing association is explained by greater indirect effects via GY (Table 5).

Table 5. Estimates of genotypic correlation coefficients and respective estimates of the direct and indirect effect of the explanatory variables based on ridge path analysis performed using values of $\mathrm{k}=0.10$ and path analysis, determination coefficient and effect of the residual variable for the 36 early maturing maize genotypes.

\begin{tabular}{|c|c|c|c|c|c|c|c|c|c|c|c|c|c|c|c|c|}
\hline \multirow[t]{3}{*}{ Effect } & \multicolumn{16}{|c|}{ Main variable } \\
\hline & \multicolumn{11}{|c|}{ Protein-nutritional } & \multicolumn{4}{|c|}{ Energetic-nutritional } & \multirow[b]{2}{*}{$\mathrm{VIF}^{1}$} \\
\hline & $\mathrm{CP}$ & Lys & Met & Cys & Thr & Trp & Val & Ile & Leu & Phe & Arg & AMEn & $\mathrm{EE}$ & $\mathrm{CF}$ & AML & \\
\hline Direct of MF on & -0.312 & -0.291 & -0.022 & -0.168 & -0.110 & -0.041 & -0.264 & -0.209 & -0.340 & -0.247 & -0.248 & -0.155 & -0.246 & -0.132 & -0.142 & 3.542 \\
\hline Indirect of $\mathrm{MF}$ via $\mathrm{FF}$ on & -0.233 & 0.198 & 0.238 & -0.029 & 0.088 & 0.102 & 0.183 & 0.211 & 0.060 & 0.133 & -0.058 & -0.125 & 0.272 & -0.718 & -0.076 & 2.820 \\
\hline Indirect of $\mathrm{MF}$ via $\mathrm{PH}$ on & 0.037 & 0.039 & -0.006 & 0.088 & -0.010 & 0.000 & 0.051 & 0.017 & 0.064 & 0.051 & 0.044 & -0.007 & 0.072 & 0.084 & -0.141 & 0.084 \\
\hline Indirect of MF via $\mathrm{EH}$ on & 0.283 & 0.149 & 0.058 & 0.153 & 0.078 & 0.053 & 0.093 & 0.042 & 0.083 & 0.072 & 0.200 & 0.252 & 0.124 & 0.052 & 0.116 & 0.266 \\
\hline Indirect of MF via EW on & 0.073 & 0.005 & -0.049 & 0.025 & 0.091 & -0.001 & 0.001 & 0.038 & 0.043 & 0.034 & 0.115 & 0.149 & -0.123 & 0.000 & -0.006 & 1.716 \\
\hline Indirect of MF via GY on & 0.218 & 0.306 & 0.124 & 0.332 & 0.308 & 0.325 & 0.302 & 0.277 & 0.343 & 0.305 & 0.323 & 0.130 & 0.167 & 0.048 & -0.068 & 1.625 \\
\hline Genotypic correlation $\left(\mathrm{r}_{\mathrm{g}}\right)$ & 0.035 & 0.377 & 0.342 & 0.384 & 0.435 & 0.434 & 0.340 & 0.355 & 0.220 & 0.324 & 0.351 & 0.228 & 0.241 & -0.679 & -0.332 & \\
\hline Direct of FF on & -0.266 & 0.226 & 0.272 & -0.034 & 0.101 & 0.117 & 0.209 & 0.241 & 0.069 & 0.152 & -0.067 & -0.143 & 0.311 & -0.821 & -0.087 & 4.458 \\
\hline Indirect of $\mathrm{FF}$ via $\mathrm{MF}$ on & -0.273 & -0.255 & -0.019 & -0.147 & -0.096 & -0.036 & -0.231 & -0.183 & -0.297 & -0.216 & -0.217 & -0.136 & -0.215 & -0.115 & -0.124 & 2.241 \\
\hline Indirect of $\mathrm{FF}$ via $\mathrm{PH}$ on & 0.039 & 0.041 & -0.006 & 0.094 & -0.010 & 0.000 & 0.054 & 0.018 & 0.068 & 0.054 & 0.047 & -0.008 & 0.077 & 0.090 & -0.150 & 0.095 \\
\hline Indirect of FF via EH on & 0.163 & 0.086 & 0.033 & 0.088 & 0.045 & 0.030 & 0.053 & 0.024 & 0.048 & 0.041 & 0.115 & 0.145 & 0.071 & 0.030 & 0.067 & 0.088 \\
\hline Indirect of $F F$ via $E W$ on & 0.092 & 0.006 & -0.062 & 0.032 & 0.115 & -0.001 & 0.002 & 0.048 & 0.055 & 0.043 & 0.145 & 0.189 & -0.156 & 0.000 & -0.008 & 2.751 \\
\hline Indirect of $\mathrm{FF}$ via $\mathrm{GY}$ on & 0.291 & 0.410 & 0.166 & 0.444 & 0.412 & 0.436 & 0.405 & 0.371 & 0.459 & 0.408 & 0.432 & 0.174 & 0.223 & 0.065 & -0.092 & 2.912 \\
\hline Genotypic correlation $\left(\mathrm{r}_{\mathrm{g}}\right)$ & 0.020 & 0.537 & 0.412 & 0.473 & 0.577 & 0.558 & 0.513 & 0.543 & 0.408 & 0.498 & 0.450 & 0.207 & 0.342 & -0.834 & -0.402 & \\
\hline Direct of PH on & 0.141 & 0.148 & -0.023 & 0.336 & -0.037 & -0.001 & 0.195 & 0.063 & 0.243 & 0.194 & 0.169 & -0.028 & 0.275 & 0.321 & -0.536 & 1.465 \\
\hline Indirect of $\mathrm{PH}$ via $\mathrm{MF}$ on & -0.082 & -0.077 & -0.006 & -0.044 & -0.029 & -0.011 & -0.069 & -0.055 & -0.089 & -0.065 & -0.065 & -0.041 & -0.065 & -0.035 & -0.037 & 0.202 \\
\hline Indirect of $\mathrm{PH}$ via $\mathrm{FF}$ on & -0.074 & 0.063 & 0.076 & -0.009 & 0.028 & 0.033 & 0.058 & 0.067 & 0.019 & 0.042 & -0.019 & -0.040 & 0.087 & -0.229 & -0.024 & 0.288 \\
\hline Indirect of $\mathrm{PH}$ via $\mathrm{EH}$ on & 0.294 & 0.155 & 0.060 & 0.159 & 0.081 & 0.055 & 0.097 & 0.044 & 0.087 & 0.075 & 0.208 & 0.262 & 0.129 & 0.054 & 0.121 & 0.287 \\
\hline Indirect of $\mathrm{PH}$ via $\mathrm{EW}$ on & -0.002 & 0.000 & 0.002 & -0.001 & -0.003 & 0.000 & 0.000 & -0.001 & -0.001 & -0.001 & -0.004 & -0.005 & 0.004 & 0.000 & 0.000 & 0.002 \\
\hline Indirect of PH via GY on & -0.011 & -0.016 & -0.007 & -0.017 & -0.016 & -0.017 & -0.016 & -0.015 & -0.018 & -0.016 & -0.017 & -0.007 & -0.009 & -0.003 & 0.004 & 0.004 \\
\hline Genotypic correlation $\left(\mathrm{r}_{\mathrm{g}}\right)$ & 0.280 & 0.289 & 0.101 & 0.457 & 0.020 & 0.058 & 0.284 & 0.110 & 0.265 & 0.249 & 0.290 & 0.139 & 0.450 & 0.140 & -0.527 & \\
\hline Direct of EH on & 0.635 & 0.335 & 0.130 & 0.343 & 0.176 & 0.118 & 0.208 & 0.095 & 0.187 & 0.161 & 0.449 & 0.565 & 0.278 & 0.117 & 0.260 & 1.615 \\
\hline Indirect of $\mathrm{EH}$ via $\mathrm{MF}$ on & -0.139 & -0.130 & -0.010 & -0.075 & -0.049 & -0.018 & -0.118 & -0.093 & -0.152 & -0.110 & -0.111 & -0.069 & -0.110 & -0.059 & -0.063 & 0.583 \\
\hline Indirect of $\mathrm{EH}$ via $\mathrm{FF}$ on & -0.068 & 0.058 & 0.070 & -0.009 & 0.026 & 0.030 & 0.054 & 0.062 & 0.018 & 0.039 & -0.017 & -0.037 & 0.080 & -0.210 & -0.022 & 0.242 \\
\hline Indirect of EH via PH on & 0.065 & 0.069 & -0.010 & 0.156 & -0.017 & -0.001 & 0.090 & 0.029 & 0.113 & 0.090 & 0.079 & -0.013 & 0.128 & 0.149 & -0.248 & 0.260 \\
\hline Indirect of $\mathrm{EH}$ via $\mathrm{EW}$ on & 0.004 & 0.000 & -0.002 & 0.001 & 0.005 & 0.000 & 0.000 & 0.002 & 0.002 & 0.002 & 0.006 & 0.008 & -0.006 & 0.000 & 0.000 & 0.005 \\
\hline Indirect of EH via GY on & 0.001 & 0.001 & 0.001 & 0.001 & 0.001 & 0.001 & 0.001 & 0.001 & 0.001 & 0.001 & 0.001 & 0.001 & 0.001 & 0.000 & 0.000 & 0.000 \\
\hline Genotypic correlation $\left(\mathrm{r}_{\mathrm{g}}\right)$ & 0.562 & 0.367 & 0.191 & 0.452 & 0.159 & 0.143 & 0.257 & 0.106 & 0.188 & 0.200 & 0.452 & 0.511 & 0.398 & 0.008 & -0.049 & \\
\hline Direct of EW on & -0.126 & -0.008 & 0.084 & -0.043 & -0.157 & 0.001 & -0.002 & -0.065 & -0.075 & -0.059 & -0.198 & -0.258 & 0.213 & 0.000 & 0.010 & 6.198 \\
\hline Indirect of EW via MF on & 0.181 & 0.169 & 0.013 & 0.098 & 0.064 & 0.024 & 0.153 & 0.121 & 0.197 & 0.143 & 0.143 & 0.090 & 0.142 & 0.076 & 0.082 & 0.981 \\
\hline Indirect of $\mathrm{EW}$ via $\mathrm{FF}$ on & 0.195 & -0.166 & -0.199 & 0.025 & -0.074 & -0.086 & -0.153 & -0.176 & -0.050 & -0.111 & 0.049 & 0.105 & -0.228 & 0.602 & 0.064 & 1.978 \\
\hline Indirect of EW via PH on & 0.003 & 0.003 & 0.000 & 0.007 & -0.001 & 0.000 & 0.004 & 0.001 & 0.005 & 0.004 & 0.003 & -0.001 & 0.005 & 0.006 & -0.011 & 0.000 \\
\hline Indirect of $\mathrm{EW}$ via $\mathrm{EH}$ on & -0.019 & -0.010 & -0.004 & -0.010 & -0.005 & -0.003 & -0.006 & -0.003 & -0.006 & -0.005 & -0.013 & -0.017 & -0.008 & -0.003 & -0.008 & 0.001 \\
\hline Indirect of EW via GY on & -0.390 & -0.549 & -0.223 & -0.595 & -0.552 & -0.583 & -0.542 & -0.497 & -0.614 & -0.547 & -0.579 & -0.233 & $\begin{array}{l}-0.0069 \\
-0.299\end{array}$ & -0.087 & 0.123 & 5.223 \\
\hline Genotypic correlation $\left(\mathrm{r}_{\mathrm{g}}\right)$ & -0.169 & -0.562 & -0.321 & -0.524 & -0.741 & -0.648 & -0.547 & -0.626 & -0.551 & -0.581 & -0.614 & -0.339 & -0.153 & 0.594 & 0.262 & \\
\hline Direct of GY on & -0.393 & -0.553 & -0.224 & -0.599 & -0.557 & -0.588 & -0.546 & -0.501 & -0.619 & -0.551 & -0.583 & -0.234 & -0.301 & -0.087 & 0.124 & 6.415 \\
\hline Indirect of GY via MF on & 0.173 & 0.161 & 0.012 & 0.093 & 0.061 & 0.023 & 0.146 & 0.116 & 0.188 & 0.137 & 0.137 & 0.086 & 0.136 & 0.073 & 0.079 & 0.898 \\
\hline Indirect of GY via FF on & 0.197 & -0.167 & -0.202 & 0.025 & -0.075 & -0.087 & -0.155 & -0.178 & -0.051 & -0.113 & 0.050 & 0.106 & -0.230 & 0.609 & 0.065 & 2.023 \\
\hline Indirect of $\mathrm{GY}$ via $\mathrm{PH}$ on & 0.004 & 0.004 & -0.001 & 0.010 & -0.001 & 0.000 & 0.006 & 0.002 & 0.007 & 0.006 & 0.005 & -0.001 & 0.008 & 0.009 & -0.016 & 0.001 \\
\hline Indirect of GY via $\mathrm{EH}$ on & -0.002 & -0.001 & 0.000 & -0.001 & 0.000 & 0.000 & -0.001 & 0.000 & 0.000 & 0.000 & -0.001 & -0.001 & -0.001 & 0.000 & -0.001 & 0.000 \\
\hline Indirect of GY via EW on & -0.125 & -0.008 & 0.084 & -0.043 & -0.156 & 0.001 & -0.002 & -0.065 & -0.074 & -0.058 & -0.197 & -0.256 & 0.211 & 0.0 & 0.010 & 5.046 \\
\hline Genotypic correlation $\left(\mathrm{r}_{\mathrm{g}}\right)$ & -0.185 & -0.619 & -0.354 & -0.575 & -0.783 & -0.710 & -0.607 & -0.677 & -0.612 & -0.635 & -0.648 & -0.324 & -0.207 & 0.594 & 0.273 & \\
\hline Determination coefficient & 0.474 & 0.524 & 0.179 & 0.596 & 0.590 & 0.481 & 0.459 & 0.454 & 0.473 & 0.461 & 0.634 & 0.383 & 0.311 & 0.768 & 0.388 & \\
\hline Residual variable & 0.725 & 0.690 & 0.906 & 0.636 & 0.641 & 0.721 & 0.735 & 0.739 & 0.726 & 0.734 & 0.605 & 0.785 & 0.830 & 0.481 & 0.782 & \\
\hline
\end{tabular}

${ }^{1} \mathrm{VIF}=$ variance inflation factor. Number of days from sowing until male flowering (MF), number of days from sowing until female flowering $(\mathrm{FF})$, plant height $(\mathrm{PH})$, ear insertion height $(\mathrm{EH})$, ear weight $(\mathrm{EW})$, and grain yield (GY) on the main variables: crude protein (CP), lysine (Lys), methionine (Met), cysteine (Cys), threonine (Thr), tryptophan (Trp), valine (Val), isoleucine (Ile), leucine (Leu), phenylalanine (Phe), and arginine (Arg), apparent metabolizable energy corrected for nitrogen (AMEn), ether extract (EE), crude fiber (CF), and amylose (AML).

In super-early maturing genotypes, MF showed a positive linear correlation $\left(\mathrm{r}_{\mathrm{g}}=\right.$ 0.581 ) and positive direct effect (direct effect $=0.491$ ) on Trp. FF showed a positive linear correlation $\left(\mathrm{r}_{\mathrm{g}}=0.725\right)$ and a positive direct effect (direct effect $\left.=0.493\right)$ on EE. PH showed a positive linear correlation $\left(r_{\mathrm{g}}=0.335\right)$ and a positive direct effect (direct effect $\left.=0.448\right)$ on CF. EH showed a positive linear correlation and positive direct effects on AMEn (direct effect $=$ 0.656 ), on EE (direct effect $=0.453$ ), and on AML (direct effect $=0.576$ ). The greatest direct negative effects were observed for the EW trait, with a negative linear correlation $(-0.878$ $\left.\leq \mathrm{r}_{\mathrm{g}} \leq-0.841\right)$ on Trp, Val, Ile, Leu, and Phe (-0.509 $\leq$ direct effect $\left.\leq-0.399\right)$. GY showed a 
negative linear correlation $\left(\mathrm{r}_{\mathrm{g}}=-0.722\right)$ and a direct negative effect (direct effect $\left.=-0.399\right)$ on Lys (Table 6).

Table 6. Estimates of genotypic correlation coefficients and respective estimates of the direct and indirect effect of the explanatory variables based on ridge path analysis performed using values of $k=0.10$ and path analysis, determination coefficient, and effect of the residual variable for the 22 super-early maturing maize genotype.

\begin{tabular}{|c|c|c|c|c|c|c|c|c|c|c|c|c|c|c|c|c|}
\hline \multirow[t]{3}{*}{ Effect } & \multicolumn{16}{|c|}{ Main variable } \\
\hline & \multicolumn{11}{|c|}{ Protein-nutritional } & \multicolumn{4}{|c|}{ Energetic-nutritional } & \\
\hline & $\mathrm{CP}$ & Lys & Met & Cys & Thr & Trp & Val & Ile & Leu & Phe & Arg & AMEn & $\mathrm{EE}$ & $\mathrm{CF}$ & AML & $\mathrm{VIF}^{1}$ \\
\hline Direct of MF on & 0.399 & 0.141 & -0.037 & 0.286 & 0.382 & 0.491 & 0.133 & 0.168 & 0.275 & 0.298 & 0.180 & -0.363 & 0.021 & 0.098 & -0.362 & 4.324 \\
\hline Indirect of MF via FF on & 0.284 & 0.056 & 0.368 & 0.342 & 0.209 & -0.054 & 0.200 & 0.265 & 0.096 & 0.156 & 0.241 & 0.451 & 0.444 & -0.150 & 0.271 & 3.619 \\
\hline Indirect of MF via PH on & -0.124 & -0.056 & -0.005 & -0.069 & -0.033 & -0.143 & 0.007 & 0.023 & -0.016 & -0.043 & -0.063 & -0.002 & -0.080 & 0.234 & -0.051 & 0.410 \\
\hline Indirect of MF via EH on & -0.352 & -0.150 & 0.052 & -0.234 & -0.351 & -0.152 & -0.196 & -0.336 & -0.351 & -0.271 & -0.050 & 0.560 & 0.387 & -0.179 & 0.491 & 2.161 \\
\hline Indirect of MF via EW on & 0.073 & 0.146 & 0.066 & 0.149 & 0.189 & 0.269 & 0.211 & 0.238 & 0.256 & 0.222 & 0.075 & -0.264 & -0.165 & 0.124 & -0.158 & 1.397 \\
\hline Indirect of MF via GY on & 0.014 & 0.218 & 0.130 & 0.118 & 0.172 & 0.121 & 0.175 & 0.144 & 0.190 & 0.153 & 0.158 & 0.098 & 0.074 & -0.090 & 0.083 & 1.609 \\
\hline Genotypic correlation $\left(\mathrm{r}_{\mathrm{g}}\right)$ & 0.334 & 0.368 & 0.571 & 0.620 & 0.606 & 0.581 & 0.542 & 0.519 & 0.478 & 0.545 & 0.559 & 0.444 & 0.683 & 0.047 & 0.239 & \\
\hline Direct of FF on & 0.316 & 0.062 & 0.409 & 0.380 & 0.232 & -0.061 & 0.222 & 0.294 & 0.107 & 0.173 & 0.267 & 0.501 & 0.493 & -0.166 & 0.301 & 5.404 \\
\hline Indirect of $\mathrm{FF}$ via $\mathrm{MF}$ on & 0.359 & 0.127 & -0.033 & 0.257 & 0.344 & 0.442 & 0.120 & 0.152 & 0.247 & 0.268 & 0.162 & -0.327 & 0.019 & 0.088 & -0.326 & 2.895 \\
\hline Indirect of FF via PH on & -0.118 & -0.053 & -0.004 & -0.066 & -0.032 & -0.137 & 0.007 & 0.022 & -0.015 & -0.041 & -0.060 & -0.002 & -0.077 & 0.224 & -0.049 & 0.375 \\
\hline Indirect of FF via EH on & -0.332 & -0.141 & 0.049 & -0.220 & -0.332 & -0.143 & -0.185 & -0.317 & -0.331 & -0.256 & -0.047 & 0.529 & 0.365 & -0.169 & 0.464 & 1.923 \\
\hline Indirect of FF via EW on & 0.101 & 0.202 & 0.091 & 0.206 & 0.262 & 0.373 & 0.292 & 0.330 & 0.355 & 0.307 & 0.104 & -0.366 & -0.229 & 0.172 & -0.218 & 2.679 \\
\hline Indirect of FF via GY on & 0.020 & 0.305 & 0.183 & 0.165 & 0.242 & 0.170 & 0.245 & 0.202 & 0.267 & 0.215 & 0.221 & 0.137 & 0.104 & -0.127 & 0.117 & 3.172 \\
\hline Genotypic correlation $\left(\mathrm{r}_{\mathrm{g}}\right)$ & 0.377 & 0.507 & 0.735 & 0.760 & 0.740 & 0.639 & 0.723 & 0.712 & 0.640 & 0.684 & 0.674 & 0.523 & 0.725 & 0.005 & 0.319 & \\
\hline Direct of PH on & -0.237 & -0.106 & -0.009 & -0.133 & -0.064 & -0.273 & 0.013 & 0.043 & -0.030 & -0.082 & -0.120 & -0.004 & -0.153 & 0.448 & -0.097 & 1.812 \\
\hline Indirect of $\mathrm{PH}$ via $\mathrm{MF}$ on & 0.209 & 0.074 & -0.019 & 0.150 & 0.200 & 0.257 & 0.070 & 0.088 & 0.144 & 0.156 & 0.094 & -0.190 & 0.011 & 0.051 & -0.190 & 0.979 \\
\hline Indirect of PH via FF on & 0.158 & 0.031 & 0.205 & 0.190 & 0.116 & -0.030 & 0.111 & 0.147 & 0.054 & 0.087 & 0.134 & 0.251 & 0.247 & -0.083 & 0.151 & 1.120 \\
\hline Indirect of $\mathrm{PH}$ via $\mathrm{EH}$ on & -0.270 & -0.115 & 0.040 & -0.179 & -0.269 & -0.116 & -0.151 & -0.258 & -0.269 & -0.208 & -0.038 & 0.430 & 0.297 & -0.137 & 0.377 & 1.271 \\
\hline Indirect of PH via EW on & 0.025 & 0.050 & 0.022 & 0.051 & 0.065 & 0.092 & 0.072 & 0.082 & 0.088 & 0.076 & 0.026 & -0.090 & -0.057 & 0.042 & -0.054 & 0.164 \\
\hline Indirect of PH via GY on & 0.005 & 0.074 & 0.044 & 0.040 & 0.059 & 0.041 & 0.060 & 0.049 & 0.065 & 0.052 & 0.054 & 0.033 & 0.025 & -0.031 & 0.028 & 0.187 \\
\hline Genotypic correlation $\left(\mathrm{rg}_{\mathrm{g}}\right)$ & -0.134 & -0.003 & 0.283 & 0.106 & 0.101 & -0.056 & 0.177 & 0.156 & 0.048 & 0.073 & 0.137 & 0.429 & 0.355 & 0.335 & 0.206 & \\
\hline Direct of EH on & -0.412 & -0.176 & 0.061 & -0.274 & -0.412 & -0.178 & -0.230 & -0.394 & -0.411 & -0.318 & -0.058 & 0.656 & 0.453 & -0.209 & 0.576 & 3.590 \\
\hline Indirect of EH via MF on & 0.340 & 0.120 & -0.032 & 0.244 & 0.326 & 0.419 & 0.114 & 0.144 & 0.234 & 0.254 & 0.154 & -0.310 & 0.018 & 0.083 & -0.309 & 2.603 \\
\hline Indirect of EH via FF on & 0.254 & 0.050 & 0.329 & 0.306 & 0.187 & -0.049 & 0.179 & 0.237 & 0.086 & 0.139 & 0.215 & 0.403 & 0.397 & -0.134 & 0.243 & 2.896 \\
\hline Indirect of $\mathrm{EH}$ via PH on & -0.0 .155 & -0.070 & -0.006 & -0.087 & -0.042 & -0.179 & 0.009 & 0.028 & -0.019 & $\frac{-1.054}{-0.054}$ & -0.079 & -0.003 & -0.100 & 0.293 & $\frac{-2.064}{-0.064}$ & 20.641 \\
\hline Indirect of $\mathrm{EH}$ via $\mathrm{EW}$ on & 0.073 & 0.146 & 0.066 & 0.149 & 0.189 & 0.270 & 0.211 & 0.239 & 0.256 & 0.222 & 0.075 & -0.264 & -0.165 & 0.124 & -0.158 & 1.401 \\
\hline Indirect of $\mathrm{EH}$ via GY on & 0.014 & 0.213 & 0.128 & 0.115 & 0.169 & 0.119 & 0.171 & 0.141 & 0.186 & 0.150 & 0.154 & 0.096 & 0.072 & -0.089 & 0.082 & 1.541 \\
\hline Genotypic correlation $(\mathrm{rg})$ & 0.073 & 0.266 & 0.552 & 0.42 & 0.377 & 0.384 & 0.430 & 0.355 & 0.291 & & & & 0.721 & 0.048 & 0.427 & \\
\hline Direct of & $\frac{0.0138}{-0.138}$ & -0.275 & $\frac{-032}{-0.124}$ & $\frac{0.427}{-0.282}$ & -0.357 & $\frac{0.004}{-0.509}$ & $\frac{-.430}{-0.399}$ & $\frac{-0.450}{-0.450}$ & $\frac{-2.21}{-0.484}$ & $\frac{0.50}{-0.4}$ & $\frac{0.43}{-0.142}$ & & 0.312 & -0.234 & & 6.029 \\
\hline Indirect of EW via MF on & -0.211 & -0.074 & 0.020 & -0.151 & -0.202 & -0.260 & -0.071 & -0.089 & -0.145 & -0.158 & -0.095 & 0.192 & -0.011 & -0.052 & 0.192 & 1.002 \\
\hline Indirect of EW via FF on & -0.231 & -0.046 & -0.300 & -0.279 & -0.170 & 0.044 & -0.163 & -0.216 & -0.078 & -0.127 & -0.196 & -0.367 & -0.362 & 0.122 & -0.221 & 2.402 \\
\hline Indirect of E & 0.04 & 0.019 & 0.0 & 0.0 & 0.0 & 0.0 & -0.002 & -0.0 & 0.005 & 0.0 & 0.022 & & 0.028 & -0.081 & & 0.049 \\
\hline Indirect of EW vi & 0.219 & 0.093 & -0.032 & 0.14 & 0.218 & 0.094 & 0.122 & 0.209 & 0.218 & 0.169 & 0.031 & -0.348 & -0.240 & 0.111 & -0.0305 & 0.834 \\
\hline Indirect of EW via & -0.026 & -0.396 & -0.237 & -0.214 & -0.314 & -0.221 & -0.318 & -0.262 & -0.346 & -0.279 & -0.287 & -0.178 & -0.135 & 0.165 & -0.152 & 5.331 \\
\hline Genotypic correlation $\left(\mathrm{r}_{\mathrm{g}}\right)$ & -0.359 & -0.706 & -0.684 & -0.785 & -0.849 & -0.852 & -0.870 & -0.861 & -0.878 & -0.841 & -0.682 & -0.152 & -0.377 & 0.008 & -0.141 & \\
\hline Direct of $\mathrm{C}$ & -0.027 & -0.399 & -0.239 & -0.216 & -0.316 & -0.222 & -0.320 & -0.264 & -0.348 & -0.281 & -0.289 & -0.180 & -0.136 & 0.166 & -0.153 & 6.556 \\
\hline Indirect of GY via MF on & -0.0217 & -0.077 & 0.020 & -0.156 & -0.208 & -0.267 & -0.073 & -0.092 & -0.150 & $\begin{array}{ll}-0.201 \\
-0.162\end{array}$ & -0.098 & 0.198 & $\begin{array}{ll}-0.150 \\
-0.011\end{array}$ & -0.053 & 0.198 & $\frac{0.050}{1.061}$ \\
\hline Indirect of GY via FF on & -0.241 & -0.048 & -0.313 & -0.291 & -0.178 & 0.046 & -0.170 & -0.225 & -0.082 & -0.132 & -0.205 & -0.383 & -0.377 & 0.127 & -0.231 & 2.615 \\
\hline Indirect of GY via PH on & 0.04 & 0.020 & 0.00 & 0.02 & 0.01 & 0.051 & -0.002 & -0.008 & 0.006 & 0.015 & 0.022 & 0.001 & 0.028 & -0.083 & 0.018 & 0.052 \\
\hline Indirect of GY via EH on & 0.220 & 0.094 & -0.033 & 0.146 & 0.220 & 0.095 & 0.123 & 0.210 & 0.219 & 0.169 & 0.031 & -0.350 & -0.242 & 0.112 & -0.307 & 0.844 \\
\hline Indirect of GY via EW on & -0.2137 & -0.273 & -0.0323 & -0.279 & -0.200 & -0.505 & -0.395 & -0.2146 & -0.480 & -0.416 & -0.140 & 0.495 & $\frac{0.242}{0.309}$ & $\frac{-0.1232}{-0.232}$ & 0.295 & 4.902 \\
\hline Genotypic correlation $\left(\mathrm{r}_{\mathrm{s}}\right.$ & -0.361 & -0.722 & -0.709 & -0.793 & -0.857 & -0.825 & -0.870 & -0.852 & -0.869 & -0.835 & -0.708 & -0.238 & -0.442 & 0.053 & -0.196 & \\
\hline Determination coefficient & 0.313 & 0.519 & 0.565 & 0.727 & 0.816 & 0.81 & 0.761 & 0.776 & 0.806 & 0.746 & 0.539 & 0.489 & 0.586 & 0.151 & 0.223 & \\
\hline Residual variable & 0.829 & 0.693 & 0.660 & 0.522 & 0.429 & 0.435 & 0.489 & 0.473 & 0.441 & 0.504 & 0.679 & 0.715 & 0.643 & 0.922 & 0.881 & \\
\hline
\end{tabular}

${ }^{1} \mathrm{VIF}=$ variance inflation factor. Number of days from sowing until male flowering (MF), number of days from sowing until female flowering $(\mathrm{FF})$, plant height $(\mathrm{PH})$, ear insertion height $(\mathrm{EH})$, ear weight $(\mathrm{EW})$, and grain yield (GY) on the main variables: crude protein (CP), lysine (Lys), methionine (Met), cysteine (Cys), threonine (Thr), tryptophan (Trp), valine (Val), isoleucine (Ile), leucine (Leu), phenylalanine (Phe), and arginine (Arg), apparent metabolizable energy corrected for nitrogen (AMEn), ether extract (EE), crude fiber (CF), and amylose (AML).

The MF, FF, and PH traits showed the greatest indirect effects via EH on AMEn and AML. MF showed an indirect effect via FF on EE (indirect effect $=0.444$ ). EH showed an indirect effect via MF on Trp (indirect effect $=0.419$ ). EW showed an indirect effect via GY on Lys (indirect effect $=-0.396$ ). GY showed an indirect negative effect via EW on Trp, Val, Ile, Leu, and Phe $(-0.505 \leq$ indirect effect $\leq-0.395)$ and positive indirect effect on AMEn (indirect effect $=0.495)($ Table 6).

There was a difference between early maturing genotypes and super-early maturing genotypes in the linear relationships and the magnitudes of the estimates of direct and indirect effects of explanatory variables on principal variables. In early maturing genotypes, FF, PH, $\mathrm{EH}$, and GY can be used in indirect selection, and in super-early maturing genotypes, this is

Genetics and Molecular Research 16 (2): gmr16029671 
true for MF, FF, PH, EH, EW, and GY. Therefore, breeders can consider indirect selection in early maturing genotypes based on higher $\mathrm{EH}$ and lower FF, PH, and GY, since these traits contribute to increasing levels of CP, Lys, Cys, Thr, Trp, Val, Ile, Leu, Phe, Arg, AMEn, CF, and AML in maize grains. In super-early maturing genotypes, selection should be based on higher MF, FF, PH, and EH, and lower EW and GY, since these traits indicate increased levels of Lys, Trp, Val, Ile, Leu, Phe, AMEn, EE, CF, and AML.

\section{CONCLUSIONS}

The number of days from sowing to male flowering, the number of days from sowing to female flowering, plant height, ear insertion height, ear weight, and grain yield can be used in indirect selection as indicators of grain nutritional quality.

\section{Conflicts of interest}

The authors declare no conflict of interest.

\section{ACKNOWLEDGMENTS}

We thank Conselho Nacional de Desenvolvimento Científico e Tecnológico (CNPq, Processes \#401372/2015-4 and \#305057/2013-8) for granting scholarships to the authors. Mr. Washington Neves of the Adisseo Brasil Company kindly conducted the laboratory tests. Student fellows and volunteers assisted in data collection.

\section{REFERENCES}

Aliu S, Rusinovci I, Fetahu S and Simeonovska E (2012). Genetic diversity and correlation estimates for grain yield and quality traits in Kosovo local maize (Zea mays L.) populations. Acta Agric. Slov. 99: 121-128. https://doi.org/10.2478/ v10014-012-0011-6

Amini Z, Khodambashi M and Houshmand S (2013). Correlation and path coefficient analysis of seed yield related traits in maize. Int. J. Agric. Crop Sci. 5: 2217-2220.

Baretta D, Nardino M, Carvalho IR, Nornberg R, et al. (2016). Path analysis for morphological characters and grain yield of maize hybrids. Aust. J. Crop Sci. 10: 1655-1661. https://doi.org/10.21475/ajcs.2016.10.12.p7707

Batal A and Dale N (2010). Ingredient Analysis Table: 2011 edition. Feedstuffs 1: 16-19.

Bekele A and Rao TN (2014). Estimates of heritability, genetic advance and correlation study for yield and it's attributes in maize (Zea mays L.). J. Plant Sci. 2: 1-4.

Bello OB, Abdulmaliq SY, Afolabi MS and Ige SA (2010). Correlation and path coefficient analysis of yield and agronomic characters among open pollinated maize varieties and their F1 hybrids in a diallel cross. Afr. J. Biotechnol. L. 9: 26332639.

Bizeti HS, Carvalho CGP, Souza JRP and Destro D (2004). Path analysis under multicollinearity in soybean. Braz. Arch. Biol. Technol. 47: 669-676. https://doi.org/10.1590/S1516-89132004000500001

Campos H (1983). Estatística experimental não-paramétrica. 4 ed. Departamento de Matemática e Estatística - ESALQ, Piracicaba.

Carvalho CGP, Oliveira VR, Cruz CD and Casali VWD (1999). Análise de trilha sob multicolinearidade em pimentão. Pesqui. Agropecu. Bras. 34: 603-613. https://doi.org/10.1590/S0100-204X1999000400011

Carvalho CGP, Borsato R, Cruz CD and Viana JMS (2001). Path analysis under multicollinearity in S0 x S0 maize hybrids. Crop Breed. Appl. Biotechnol. 1: 263-270. https://doi.org/10.13082/1984-7033.v01n03a06

Chukwu SC, Ekwu LG, Onyishi GC, Okporie EO, et al. (2015). Correlation between agronomic and chemical characteristics of maize (Zea mays L.) genotypes after two years of mass selection. Int. J. Sci. Res. 4: 1708-1712.

Coimbra JLM, Benin G, Vieira EA, Oliveira AC, et al. (2005). Consequências da multicolinearidade sobre a análise de

Genetics and Molecular Research 16 (2): gmr16029671 
trilha em canola. Cienc. Rural 35: 347-352. https://doi.org/10.1590/S0103-84782005000200015

Cruz CD (2013). GENES - a software package for analysis in experimental statistics and quantitative genetics. Acta Sci. Agron. 35: 271-276. https://doi.org/10.4025/actasciagron.v35i3.21251

Cruz CD, Regazzi AJ and Carneiro PCS (2012). Modelos biométricos aplicados ao melhoramento genético. Viçosa, MG, UFV.

El-Taweel AMSA, El-Koomy MBA and Fares WM (2012). Path analysis to control the multicollinearity among yield components in maize (Zea mays L.). Egypt. J. Agron. 34: 213-226.

Fabijanac D, Varga B, Svečnjak Z and Grbeša D (2006). Grain yield and quality of semifl int maize hybrids at two sowing dates. Agric. Conspec. Sci. 71: 45-50.

Filipović M, Babić M, Delić N, Bekavac G, et al. (2014). Determination relevant breeding criteria by the path and factor analysis in maize. Genetika 46: 49-58. https://doi.org/10.2298/GENSR1401049F

Haghi Y, Boroomandan P, Moradin M, Hassankhali M, et al. (2012). Correlation and path analysis for yield, oil and protein contente of Soybean (Glycine max L.) genotypes under different levels of nitrogen starter and plant density. Biharean Biol. 6: 32-37.

Hair JF, Black WC, Babin BJ, Anderson RE, et al. (2009). Análise multivariada de dados. 6 ed. Bookman, Porto Alegre.

Hasjim J, Srichuwong S, Scott MP and Jane JL (2009). Kernel composition, starch structure, and enzyme digestibility of opaque-2 maize and quality protein maize. J. Agric. Food Chem. 57: 2049-2055. https://doi.org/10.1021/jf803406y

Idikut L, Atalay AI, Kara SN and Kamalak A (2009). Effect of hybrid on starch, protein and yields of maize grain. Int. J. Anim. Vet. 8: 1945-1947.

Li Q, Zang J, Liu D, Piao X, et al. (2014). Predicting corn digestible and metabolizable energy content from its chemical composition in growing pigs. J. Anim. Sci. Biotechnol. 5: 11. https://doi.org/10.1186/2049-1891-5-11

Lovatto PA, Mallmann CA, Ceccantini M, Hauschild L, et al. (2006). Relations entre les caractéristiques agronomiques, nutritionnelles et mycotoxicologiques de différents hybrides de maïs. J. Rech. Porcine. 38: 163-170.

Mahesh N, Wali MC, Gowda MVC, Motagi BN, et al. (2013). Correlation and path analysis of yield and kernel components in maize. Karnataka J. Agric. Sci. 26: 306-307.

Martinez C and Cuevas-Perez F (1989). Evaluación de lacalidad culinária y molineradel arroz. $3^{\mathrm{a}}$ ed. CIAT, Cali.

Moreira SO, Gonçalves LSA, Rodrigues R, Sudré CP, et al. (2013). Correlações e análise de trilha sob multicolinearidade em linhas recombinadas de pimenta (Capsicum annuum L.). Rev. Bras. Ciên. Agr. 8: 15-20.

Mustafa HSB, Aslam M, Hasan E, Hussain F, et al. (2014). Genetic variability and path coefficient in maize (Zea mays L.) genotypes. J. Agric. Sci. 9: 37-43.

Resende MDV and Duarte JB (2007). Precisão e controle de qualidade em experimentos de avaliação de cultivares. Pesqui. Agropecu. Trop. 37: 182-194.

Saleem M, Ahsan M, Aslam M and Majeed A (2008). Comparative evaluation and correlation estimates for grain yield and quality attributes in maize. Pak. J. Bot. 40: 2361-2367.

Silva AF, Sediyama T, Silva FCS, Bezerra ARG, et al. (2015). Correlation and path analysis of soybean yield components. Int. J. Pl. An. Env. Sci. 5: 177-179.

Silva AR, Nascimento M, Cecon PR, Sapucay MJLC, et al. (2013). Path analysis in multicollinearity for fruit traits of pepper. Idesia 31: 55-60. https://doi.org/10.4067/S0718-34292013000200008

Srećkov Z, Boćanski J, Nastasić A, Đalović I, et al. (2010). Correlation and path coefficient analysis of morphological traits of maize (Zea mays L.). Res. J. Agric. Sci. 42: 292-296.

Steel RGD, Torrie JH and Dickey DA (1997). Principles and procedures of statistics: a biometrical approach. 3.ed. McGraw Hill Book, New York.

Toebe M and Cargnelutti Filho A (2013a). Multicollinearity in path analysis of maize (Zea mays L). J. Cereal Sci. 57: 453462. https://doi.org/10.1016/j.jcs.2013.01.014

Toebe M and Cargnelutti Filho A (2013b). Não normalidade multivariada e multicolinearidade na análise de trilha em milho. Pesqui. Agropecu. Bras. 48: 466-477. https://doi.org/10.1590/S0100-204X2013000500002

Torres FE, Teodoro PE, Ribeiro LP, Correa CCG, et al. (2015). Correlations and path analysis on oil content of castor genotypes. Biosci. J. 31: 1363-1369. https://doi.org/10.14393/BJ-v31n5a2015-26391

Wali MC, Salimath PM, Prashanth M and Harlapur SI (2006). Studies on character association as influenced by yield, starch and oil in maize (Zea mays L.). Karnataka J. Agric. Sci. 19: 932-935.

Wright S (1921). Correlation and causation. J. Agric. Res. 20: 557-585.

Zdunić Z, Nastasić A, Jocković Đ, Ivanović M, et al. (2012). Genetic analysis of grain yield and oil content in two maize populations. Period. Biol. 114: 67-72.

Genetics and Molecular Research 16 (2): gmr16029671 